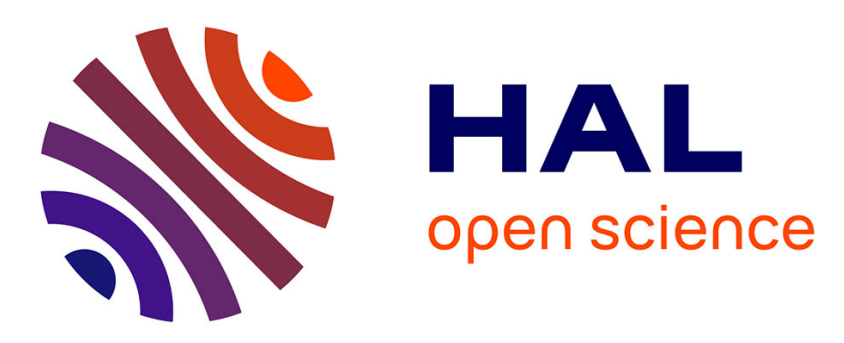

\title{
An agile electronically scanned EBG matrix antenna for monitoring target activity
}

\author{
Hussein Abou Taam, Ali Siblini, Georges Zakka El Nashef, Bernard Jecko, \\ Eric Arnaud, Nicolas Chevalier, Mohamed Rammal
}

\section{- To cite this version:}

Hussein Abou Taam, Ali Siblini, Georges Zakka El Nashef, Bernard Jecko, Eric Arnaud, et al.. An agile electronically scanned EBG matrix antenna for monitoring target activity. Joint European Radar Conference (EuRAD) with European Microwave Integrated Circuits Conference (EuMIC) and European Microwave Conference (EuMC), Sep 2015, Paris, France. pp.413-416, 10.1109/EuRAD.2015.7346325 . hal-01321026

\section{HAL Id: hal-01321026 \\ https://hal.science/hal-01321026}

Submitted on 24 May 2016

HAL is a multi-disciplinary open access archive for the deposit and dissemination of scientific research documents, whether they are published or not. The documents may come from teaching and research institutions in France or abroad, or from public or private research centers.
L'archive ouverte pluridisciplinaire HAL, est destinée au dépôt et à la diffusion de documents scientifiques de niveau recherche, publiés ou non, émanant des établissements d'enseignement et de recherche français ou étrangers, des laboratoires publics ou privés. 


\section{An Agile Electronically Scanned EBG Matrix Antenna For Monitoring Target Activity}

\author{
Hussein Abou Taam ${ }^{1,2}$, Ali Siblini ${ }^{1,2}$, Georges Zakka \\ El Nashef ${ }^{1}$, Bernard Jecko ${ }^{1}$, Eric Arnaud ${ }^{1}$, Nicolas \\ Chevalier $^{1}$ \\ XLIM OSA (Ondes et Systèmes Associés) \\ Limoges University \\ Limoges, France
}

\author{
Mohamed Rammal ${ }^{2}$ \\ GRIT Dept. \\ Lebanese University \\ Beirut, Lebanon
}

\begin{abstract}
Telecommunications, civil and military radars, RFID (Radio Frequency IDentification) and all the radiating systems are evolving towards the spatial agility that associates « range » and « coverage ». In this context, this article describes and demonstrates an experimental prototype to show the reliability and efficiency of the EBG matrix antenna theoretical aspect, for beam forming and beam steering applications.
\end{abstract}

Keywords-Beam forming; Beam steering; Electromagnetic band gap antenna; high scanning angles.

\section{INTRODUCTION}

Today's increase of functions, improvement of performances and cost reductions required on an agile electronically scanned antenna, drive researchers to develop an innovative antennas' concept in order to deal with the proposed challenge. This evolution is natural since current applications still rely on mechanical antennas that do not offer the agility and the compactness that are required for future market needs, unlike active electronically scanned array (AESA). Moreover, the main issue remains in the cost of such complex system where the market trend is to produce an innovative radiating system with low cost and excellent performances.

Therefore, in the framework of a tracking agile antenna applications (e.g. monitoring and guiding patients at hospitals), the challenge is to design and construct an efficient low cost antenna system in order to obtain an agile beam forming and steering. The proposed antenna working principle is based on the equivalent radiating surface approach and used a low profile electromagnetic band gap antenna with the implementation of an elegant beam forming network (BFN), in order to form and electronically steer the radiation pattern.

\section{ANTENNA THEORETICAL CONCEPT AND DESIGN}

The concept of the proposed antenna constitutes the subject of an accepted C.N.R.S (Centre National de la Recherche Scientifique) patent [1]. The theoretical working principle of the matrix antenna is described in details in [1] and [2]. Briefly, it is based on the equivalent radiating surface approach to overcome some of the array approach defects. The concept of the matrix antenna relies on the creation of a radiating surface presenting electromagnetic fields $\left(\mathrm{E}_{\mathrm{s}}, \mathrm{H}_{\mathrm{s}}\right)$ which can spatially vary in modulus and in phase to introduce agility on radiation patterns. The radiating surface is generated using small and jointed elementary radiating surfaces. These elementary surfaces are built by using special electromagnetic sources called 'Pixels'. The pixel should have particular electromagnetic properties in order to enhance the antenna efficiency. For this aim, the pixel's concept is inspired from the EBG antenna.

Therefore, the called matrix antenna is formed by an association of several joint and identical EBG pixels. Each EBG pixel is similar to a classical EBG antenna, acting as a resonant cavity formed between a ground plane and a Frequency Selective Surface (FSS) placed above it in the $\mathrm{z}$ direction (Fig. 1). In addition, a classical EBG antenna usually generates on its roof a circular radiating surface [2]. The original idea, in the EBG pixel, was to transform this circular radiating surface to a square radiating surface with a uniform electric field distribution. This was done by inserting four metallic walls inside the EBG cavity (Fig. 1). The presence of an evanescent mode existing into the EBG cavity, prohibiting any undesirable transverse resonance, permitted the metallic walls insertion. All these factors contributed to form a special EBG pixel with an original radiating surface which gives the matrix many electromagnetic advantages in comparison with an antenna array. We cite among them [2]: the low mutual coupling, better conservation of the gain regardless the high scanning angle values, better radiating surface efficiency and etc.

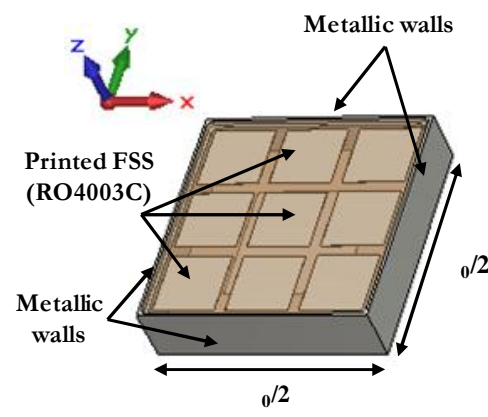

Fig. 1. EBG pixel Design. 


\section{MANUFACTURED MATRIX ANTENNA}

In the framework of a specific monitoring application, it is important to determine the specifications of the EBG matrix antenna which will be designed and manufactured. All these specifications are critical in order to determine the BFN and its components. The EBG matrix antenna must have a sufficient gain (Gain $210 \mathrm{~dB}$ ) in order to detect the specified targets beyond $100 m$.The desired gain enable us to fix the pixels number at 9. These pixels are associated along a single direction by adjacent walls, and they have an inter-element spacing of $0.5 \lambda_{0}$. Moreover, the choice of the corresponding radiofrequency technology $(\mathrm{Wi}-\mathrm{Fi})$ permits to define the working frequency of the global system $(2.48 \mathrm{GHz})$. The experimental prototype of $1 \mathrm{D}$ EBG matrix antenna is presented in Fig. 2.

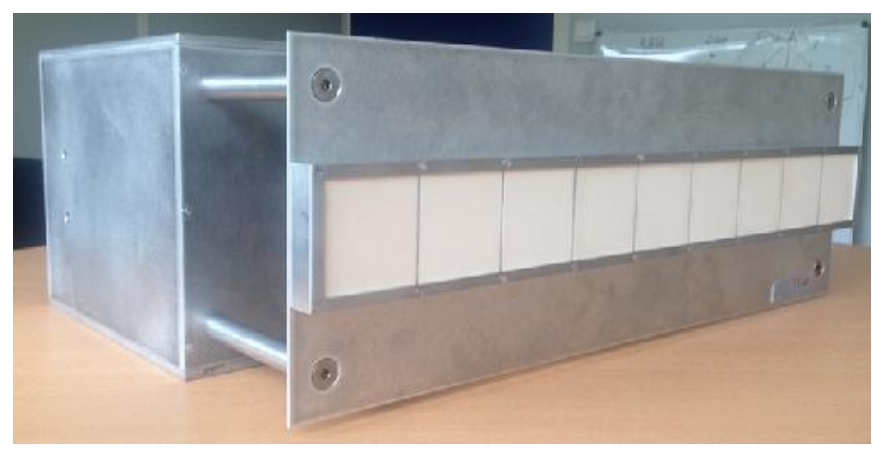

Fig. 2. Matrix antenna manufactured prototype.

The matrix prototype is fixed on a metallic box by the means of 4 metallic spacers. This metallic box should hold the BFN and should be used to fix the global system on the anechoic chamber support.

\section{BEAM FORMING NETWORK}

According to the expected specifications, the EBG matrix antenna should form a Gaussian beam having an important gain $(\geq 12 \mathrm{~dB})$ with a low side lobe levels $(\leq-20 \mathrm{~dB})$. In addition, the formed beam should cover all the angles between $-50^{\circ}$ and $+50^{\circ}$ with $10^{\circ}$ window step to monitor, detect and identify the cooperative moving targets into an angular range of $\left[-60^{\circ} ;+60^{\circ}\right]$. The BFN is composed of a power divider, digital phase shifters and an electronic control board.

\section{A. Power divider}

One of the requirements is to obtain a low side lobe levels, i.e. below or equal to $-20 \mathrm{~dB}$. In order to achieve that, a magnitude law of excitation, which enables us to obtain an important gain with low side lobe levels, is used. The excitation law was developed at XLIM laboratory and does not fit for discussion in this article. The subject of this paragraph is to show the manufactured power divider ( 1 to 9 ways) which is non equi-modulus and equi-phase.

The power divider has been designed and manufactured at 2.48 GHz using Momentum from Agilent Advanced System Design (ADS). Fig. 3 presents the manufactured power divider leading to obtain a low side lobe levels.

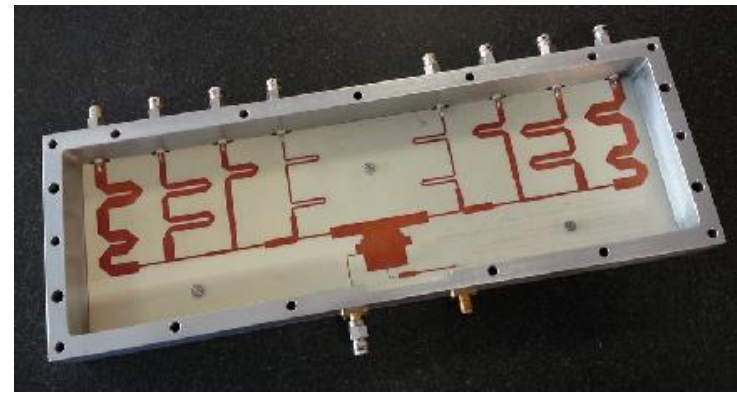

Fig. 3. A manufactured non equi-modulus and equi-phase power divider.

\section{B. Phase shifters and elecrtonic control board}

In order to scan the formed radiation beam between $\left[-50^{\circ}\right.$; $+50^{\circ}$ ], 9 commercial digital phase shifters from Macom Technology Solutions (MAPS-010164) were chosen because such phase shifters are ideally suited for use where high phase accuracy with minimum loss variation over the phase shift range are required. The phase shifters have been integrated and assembled in a metallic box (Fig. 4).

The control of the phase shifters and the generation of a correct phase at each pixel access corresponding to a steered direction are established by an electronic board. The board is composed of LCD (Liquid Crystal Display), subD 62 pins, six IC (Integrated Circuit) "ATEMGA 328P" and a receiver (Fig. 8). The LCD is connected to "Arduino UNO R3" which is based on ATEMGA $328 \mathrm{P}$. An algorithm was implemented into the IC related to the LCD in order to control the phase shifting (Fig. 5).

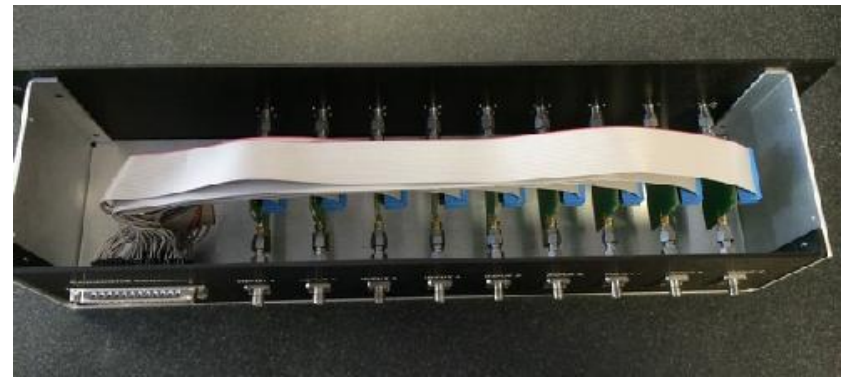

Fig. 4. Metallic box containing 9 embedded digital phase shifters.

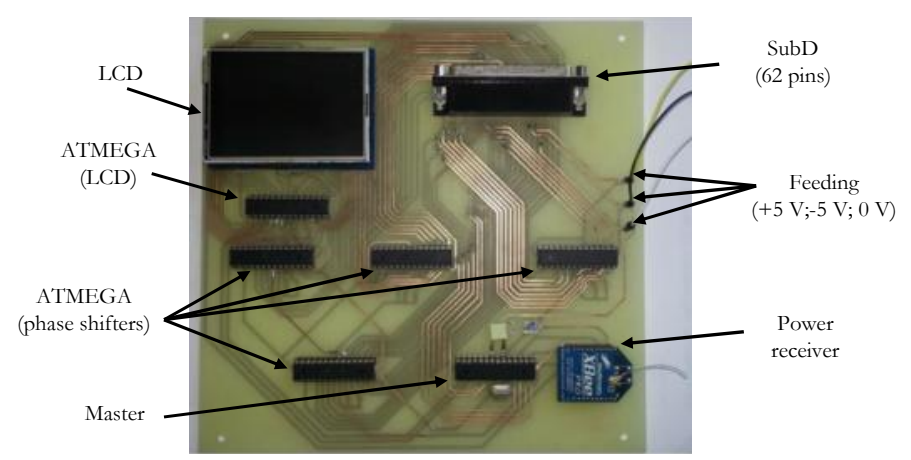

Fig. 5. Manufactured electronic control board. 


\section{COMPLETE DEMONSTRATOR}

After presenting the EBG matrix antenna and the various components of the BFN, Fig. 6 depicts the complete demonstrator. The first electromagnetic (EM) evaluations of such system were established in the anechoic chamber.

Fig. 7 presents the comparison between the simulated and the measured global matching coefficient. The results present a good agreement and exhibit a bandwidth between $[2.45 \mathrm{GHz}-$ $2.51 \mathrm{GHz}]$.

The evaluation of the EM radiation performances of the EBG matrix antenna in the presence of the BFN were carried out in two phases: broadside and steered patterns. Concerning the broadside direction, the comparison in Fig. 8.a shows a good agreement for the working frequency $(2.48 \mathrm{GHz})$. It will be important to notice that the agreement is also obtained for other frequencies of the bandwidth. The main lobe is maintained and the side lobe levels are below than $-20 \mathrm{~dB}$ (consistent with the fixed specifications).

Concerning the steered patterns, Figures 8.b and 8.c present the comparison between measured steered patterns and simulated ones respectively for $-50^{\circ}$ and $40^{\circ}$. A good agreement is observed, the same agreement is obtained as well for the remaining steered direction between $\left[-50^{\circ} ;+50^{\circ}\right]$. The steered lobes are maintained and the side lobe levels are below than $-20 \mathrm{~dB}$ as well. The shown comparison, Fig. 8, for the steered patterns was made at $2.48 \mathrm{GHz}$. However, the radiation performances, i.e. correct steering and low side lobe levels, are almost maintained over a frequency bandwidth $[2.45 \mathrm{GHz}-$ $2.51 \mathrm{GHz}]$

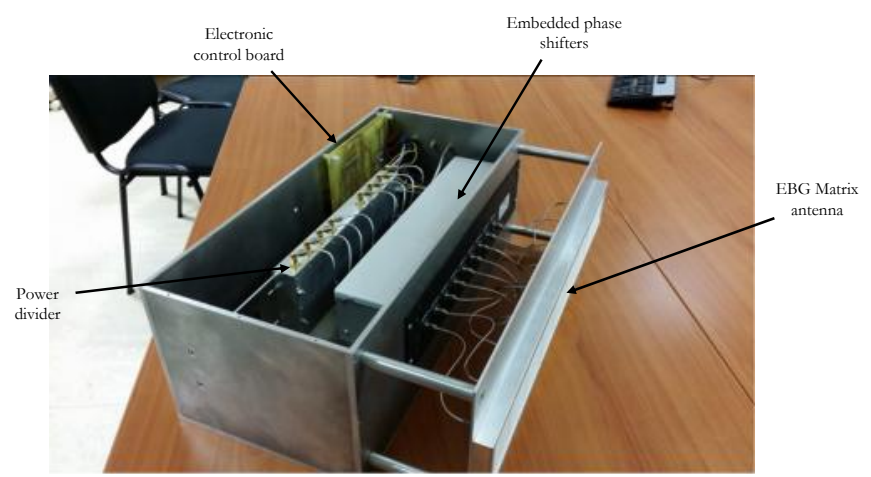

Fig. 6. Complete manufactured demonstrator : EBG Matrix antenna associated to the BFN.

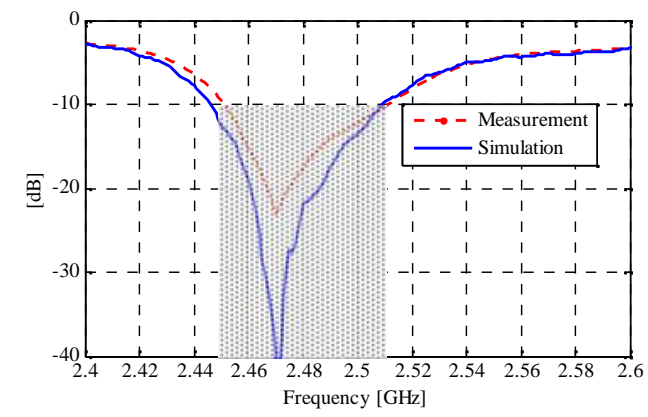

Fig. 7. Comparison simulation / measurement between the global matching coeffecient of the complet demonstrator.

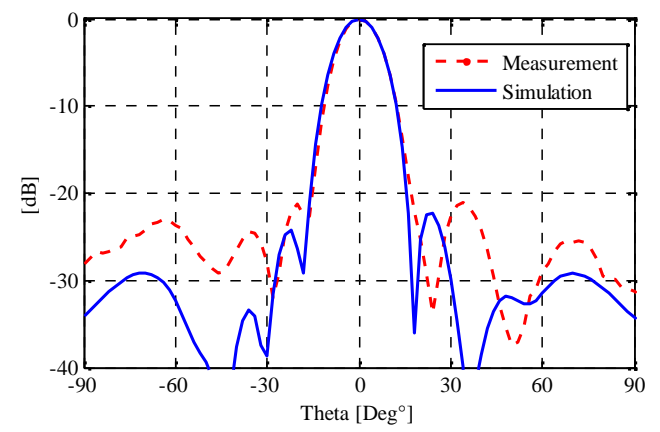

(a)

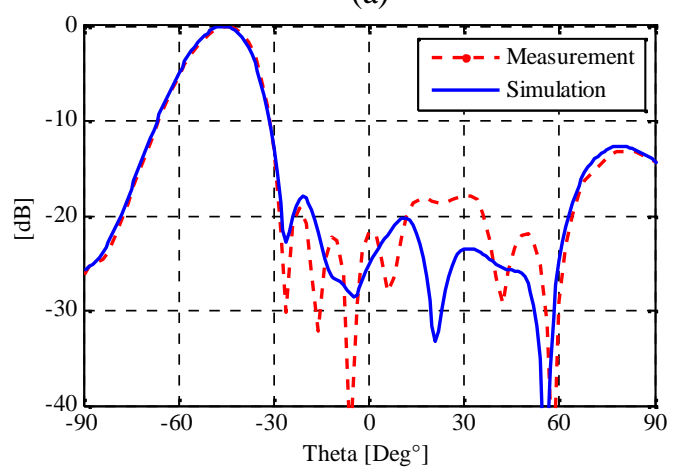

(b)

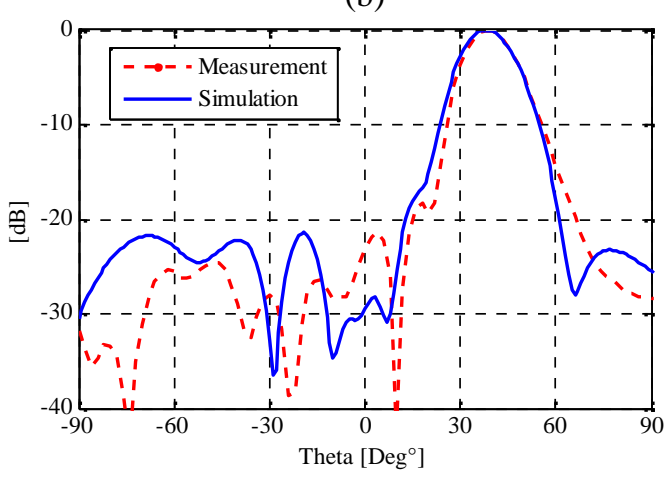

(c)

Fig. 8. Comparison simulation / measurement between the radiation patterns (a) $0^{\circ}$ (b) $-50^{\circ}$ (c) $+40^{\circ}$, at $2.48 \mathrm{GHz}$.

\section{CONCLUSION}

In this article, the EBG matrix antenna theory was briefly described. Moreover, the EBG pixel, due to the original radiating aperture, gives the matrix (anonymous jointed pixels) many EM advantages, which was demonstrated theoretically, in [2]. An experimental demonstrator composed of the EBG matrix antenna and a BFN (electronic control board + digital phase shifters + power divider) was manufactured to validate the EBG matrix antenna beam forming and beam steering capabilities for a predefined tracking application. The measurement were done in anechoic chamber and showed a very good agreement in comparison with the simulation. The theoretical concept of the EBG matrix antenna was validated experimentally in terms of radiation patterns providing an elegant, efficient and robust antenna system dedicated for agile antenna applications. 


\section{REFERENCES}

[1] Jecko, B.; Hajj, M.; Chantalat, R.; Salah Toubet, M., "Antenne élémentaire et antenne réseau mono ou bidimensionnelle correspondante," PCT Patent: PCT/EP2012/076509; French Patent: FR 1162141
[2] Hussein Abou Taam, Moustapha Salah Toubet, Thierry Monediere, Bernard Jecko and Mohammad Rammal, "A New Agile Radiating System Called Electromagnetic Band Gap", International Journal of Antennas and Propagation, Vol. 2014, Article ID 309728, 7 pages, 2014. 